\title{
Roles of oxidative stress-responsive apoptosis inducing protein (ORAIP) in diabetic complications
}

\section{Seko $\mathrm{Y}^{*}$}

Department of Biofunctional Microbiota, Graduate School of Medicine, Juntendo University, Bunkyo-ku, Tokyo 113-8421, Japan

\section{Introduction}

Oxidative stress has been strongly implicated in the pathogenesis of hyperglycemia-induced cell injury that causes various tissue damage known as diabetic complications [1]. These include microangiopathy, atherosclerotic macroangiopathy, muscle injury, and immune abnormality. It is also known that hyperglycemia induces pancreatic $\beta$-cell apoptosis leading to insulin deficiency [2]. There have been four main molecular mechanisms implicated in the hyperglycemia-induced cell injury, such as increased polyol pathway flux, increased advanced glycation end-product (AGE) formation, activation of protein kinase $\mathrm{C}$ (PKC) isoforms, and increased hexosamine pathway flux. It was proposed that all of these mechanisms reflect a single hyperglycemiainduced process, overproduction of superoxide by the mitochondrial electron-transport chain [3]. Oxidative stress induces reactive oxygen species (ROS) production, lipid peroxidation, protein oxidation and DNA damage in the cells that leads to apoptosis. Until recently, ROS have been proposed as the key mediator of oxidative stress-induced cell injury [4-6]. However, large scale antioxidants (including vitamins, free radical scavengers) clinical trials have been unsuccessful to improve the outcome of cardiovascular and cerebrovascular diseases in humans $[7,8]$ raising a possibility that there is some unknown mechanism other than ROS that mediates oxidative stress-induced cell injury.

We formerly identified a novel apoptosis-inducing humoral factor in a conditioned medium from cardiac myocytes subjected to hypoxia/ reoxygenation. In 2015, we reported that this novel secreted form of eukaryotic translation initiation factor 5A (eIF5A) is sulfated at 69th tyrosine residue and contains more hypusinated isoform than cytosolic eIF5A [9]. We found that eIF5A undergoes tyrosine-sulfation in the trans-Golgi and is rapidly secreted from cardiac myocytes in response to hypoxia/reoxygenation, then, induces apoptosis in the cells by acting as a pro-apoptotic ligand in an autocrine fashion (Figure 1). We named this novel tyrosine-sulfated secreted form of eIF5A, Oxidative stressResponsive Apoptosis Inducing Protein (ORAIP) [9]. We confirmed that ORAIP is secreted in response specifically to the oxidative stresses including ischemia/reperfusion, hypoxia/reoxygenation, ultraviolet light, ionizing radiation, cold/warm-stress (heat shock), and blood acidification [10], then plays a pivotal role in inducing apoptosis in target cells such as cardiac myocytes, and cerebral neurons. Furthermore, rat myocardial ischemia/reperfusion (but not ischemia alone) rapidly and markedly increased plasma levels of ORAIP, which returned to the control levels within $60 \mathrm{~min}$. In vivo treatment with anti-ORAIP neutralizing monoclonal antibodies (mAbs) significantly reduced myocardial ischemia/reperfusion injury [9]. These results strongly suggested that ORAIP is a specific biomarker and a critical therapeutic target for oxidative stress-induced cell injury. We also found that the plasma levels of ORAIP were markedly elevated in patients with

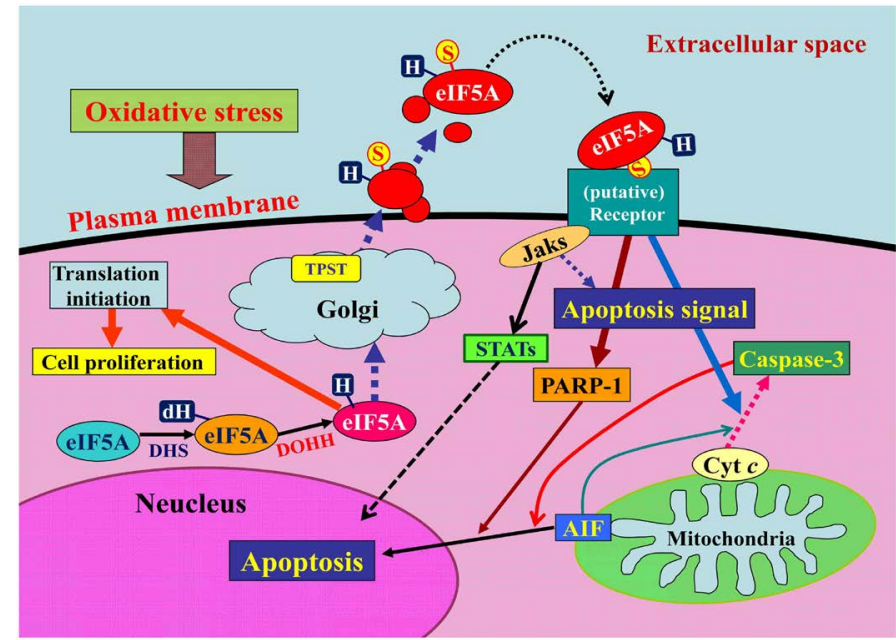

Figure 1. A model for the mechanism by which oxidative stress induces apoptosis via the autocrine secretion of eIF5A (ORAIP). (reproduced from Seko Y, et al. Sci Rep 5, 13737, doi 10.1038srep13737 (2015)

chronic diseases such as chronic kidney disease, atrial fibrillation, heart failure, dyslipidemia, diabetes mellitus, and so on, in which oxidative stress plays a critical role in the pathogenesis involved [11-13]. It seems that ORAIP may be a common humoral factor among various cell types as the dominant apoptosis-inducer in response to various types of oxidative stress. Especially, (cardiac and skeletal) myocytes, neurons, and cancer cells need a lot of oxygen for their activities, making them to be very sensitive to oxygen concentrations and hence susceptible to oxidative stress-induced apoptosis mediated by ORAIP. We previously reported that plasma ORAIP levels in diabetes model rats were about 18 times elevated during diabetic phase as compared with non-diabetic control phase, and that there was a significant positive correlation between plasma levels of glucose and ORAIP [14]. This strongly suggested that hyperglycemia plays a critical role in increasing plasma levels of ORAIP. Here, I summarize the knowledge regarding the possible roles of ORAIP in diabetic complications.

${ }^{*}$ Correspondence to: Yoshinori Seko, MD, $\mathrm{PhD}$, Department of Bio functional Microbiota, Graduate School of Medicine, Juntendo University, Bunkyo-ku, Tokyo 113-8421, Japan, Tel: +81-3-5802-1591, Fax: +81-3-3813-5512, Email: sekoyosh-tky@umin.ac.jp

Key words: atherosclerotic macroangiopathy, hyperglycemia, microangiopathy, oxidative stress, oxidative stress-responsive apoptosis inducing protein (ORAIP), reactive oxygen species (ROS)

Received: July 09, 2018; Accepted: July 19, 2018; Published: July 23, 2018 


\section{Microangiopathy}

Diabetic microangiopathy is known to cause nephropathy, retinopathy, and neuropathy. As the mechanism that capillary endothelial cells and glomerular mesangial cells are preferentially injured by hyperglycemia, it was proposed that these cells cannot reduce the transport of glucose inside the cell when they are exposed to hyperglycemia, [15]. Although we do not have the data whether ORAIP pathway is activated in these cells in response to hyperglycemia, we cannot exclude a possibility that ORAIP plays at least a partial role in diabetic microangiopathy. We previously reported that plasma levels of ORAIP were markedly elevated in dialysis patients largely with diabetic nephropathy, suggesting the roles of ORAIP in cardiovascular injury and sarcopenia in these patients [11]. Recently, we found that vitreous body concentrations of ORAIP were significantly increased in diabetic retinopathy suggesting that ORAIP plays a role in the oxidative stress-induced retinal cell injury (unpublished data). Because neurons are sensitive to oxygen concentrations, elevated plasma levels of ORAIP induced by hyperglycemia should injure peripheral neurons.

\section{Atherosclerotic macroangiopathy}

It is known that diabetes mellitus (DM) often associates hypertriglyceridemia due to insulin resistance and hyperglycemiainduced oxidative stress facilitates low density lipoprotein (LDL)cholesterol oxidation, resulting in the development of atherosclerotic macroangiopathy, which involves coronary, cerebral, and peripheral arteries. These complications cause life-threatening conditions such as ischemic heart disease, stroke, and arteriosclerosis obliterans. We found that cardiac myocytes and cerebral neurons secrete ORAIP in response to ischemia/reperfusion, which in turn induces apoptosis of the cells in an autocrine fashion. In vivo administration of a neutralizing antiORAIP mAb markedly reduced ischemia/reperfusion injury, indicating that ORAIP plays a pivotal role and will be a critical therapeutic target for ischemia/reperfusion injury induced by reperfusion therapy for acute myocardial infarction and acute ischemic stroke. Furthermore, we found co-localization of ORAIP and oxidized LDL in the plaque lesion of coronary arteries, suggesting a critical role of ORAIP in the development of plaque vulnerability leading to acute myocardial infarction (unpublished data). These findings offer a possible antiORAIP therapy for these complications.

\section{Muscle injury}

$\mathrm{DM}$ is also known to develop myocardial injury that causes various types of arrhythmia and heart failure such as diabetic cardiomyopathy, as well as sarcopenia. We reported that high glucose induced massive apoptosis in cardiac myocytes, which was mostly suppressed by neutralizing anti-ORAIP $\mathrm{mAb}$, indicating the pivotal role of ORAIP in hyperglycemia-induced myocardial injury [14]. Because cardiac and skeletal myocytes are susceptible to oxidative stress-induced apoptosis mediated by ORAIP, hyperglycemia-induced high plasma concentrations of ORAIP may play a critical role in the development of sarcopenia as well.

\section{Immune abnormality}

Although susceptibility to infection is often associated with DM patients, the precise mechanism of impairment of immune function has been still elusive. We found the strong expression of ORAIP in infiltrating immune cells such as T-cells, natural killer cells, and macrophages at the site of inflammation. This suggests that ORAIP may play a role in inducing apoptosis in target cells as well as immune cells themselves (unpublished data).

\section{Pancreatic $\beta$-cell injury}

Insulin resistance or insufficient insulin secretion have been implicated in the pathogenesis of type 2 DM. Evidence has accumulated that loss of pancreatic $\beta$-cells through hyperglycemiainduced apoptosis plays a critical role in the progression of type 2 DM [16]. We found that pancreatic $\beta$-cells strongly expressed ORAIP under hyperglycemia and recombinant ORAIP induced massive apoptosis in pancreatic $\beta$-cells in vitro [14]. This suggested that ORAIP also plays a critical role in the progression of type $2 \mathrm{DM}$ itself as well as diabetic complications.

In conclusion, increasing evidence has shown that ORAIP plays a pivotal role in oxidative stress-induced cell injury leading to diabetic complications as well as aggravating DM itself. I hope future clinical application of the anti-ORAIP therapy (such as neutralizing $\mathrm{mAb}$ ) will have innovative effects on these disorders.

\section{Conflicts of interest}

None.

\section{References}

1. Giacco F, Brownlee M (2010) Oxidative stress and diabetic complications. Circ Res 107: 1058-1070. [Crossref]

2. Tomita T (2016) Apoptosis in pancreatic $\hat{\mathrm{I}}^{2}$-islet cells in Type 2 diabetes. Bosn J Basic Med Sci 16: 162-179. [Crossref]

3. Brownlee M (2001) Biochemistry and molecular cell biology of diabetic complications Nature 414: 813-820. [Crossref]

4. Chien CT, Lee PH, Chen CF, Ma MC, Lai MK, et al. (2001) De novo demonstration and co-localization of free-radical production and apoptosis formation in rat kidney subjected to ischemia/reperfusion. J Am Soc Nephrol 12: 973-982. [Crossref]

5. Kaminski KA, Bonda TA, Korecki J, Musial WJ (2002) Oxidative stress and neutrophil activation--the two keystones of ischemia/reperfusion injury. Int J Cardiol 86: 41-59. [Crossref]

6. Madamanchi NR, Vendrov A, Runge MS (2005) Oxidative stress and vascular disease Arterioscler Thromb Vasc Biol 25: 29-38. [Crossref]

7. Vogiatzi G, Tousoulis D, Stefanadis C (2009) The role of oxidative stress in atherosclerosis. Hellenic J Cardiol 50: 402-409. [Crossref]

8. Myung SK, Ju W, Cho B, Oh SW, Park SM, et al. (2013) Efficacy of vitamin and antioxidant supplements in prevention of cardiovascular disease: systematic review and meta-analysis of randomised controlled trials. $\mathrm{Br}$ Med J 346: f10.

9. Seko Y, Fujimura T, Yao T, Taka H, Mineki R, et al. (2015) Secreted tyrosine sulfatedeIF5A mediates oxidative stress-induced apoptosis. Sci Rep 5: 13737.

10. Yao T, Fujimura T, Murayama K, Seko Y. (2016) Plasma levels of oxidative stressresponsive apoptosis inducing protein (ORAIP) in rats subjected to various types of oxidative stress. Bioscience Rep 36: $\mathrm{e} 00317$

11. Tanaka K, Yao T, Fujimura T, et al. (2016) Marked elevation of plasma levels of oxidative stress-responsive apoptosis inducing protein in dialysis patients. Kidney Int Rep 1: 321-324

12. Yao T, Tanaka K, Fujimura T, Murayama K, Fukuda S, et al. (2016) Plasma levels of oxidative stress-responsive apoptosis inducing protein (ORAIP) in patients with atrial fibrillation. Int J Cardiol 222: 528-530. [Crossref]

13. Tanaka K, Yao T, Sato K, Okumura K, Seko Y (2017) Oxidative stress-responsive apoptosis inducing protein (ORAIP) plays a critical role in the cardiac injury in patients with heart failure. Ann Pharmacol Pharm 2: 1100.

14. Yao T, Fujimura T, Murayama K, Okumura K, Seko Y. (2017) Oxidative stressresponsive apoptosis inducing protein (ORAIP) plays a critical role in high glucoseinduced apoptosis in rat cardiac myocytes and murine pancreatic $\beta$-cells. Cells 6: 35. 
Seko Y (2018) Roles of oxidative stress-responsive apoptosis inducing protein (ORAIP) in diabetic complications

15. Brownlee M (2005) The pathobiology of diabetic complications: a unifying mechanism. Diabetes 54: 1615-1625. [Crossref]
16. Tomita T (2016) Apoptosis in pancreatic $\hat{\mathrm{I}}^{2}$-islet cells in Type 2 diabetes. Bosn J Basic Med Sci 16: 162-179. [Crossref]

Copyright: $(02018$ Seko Y. This is an open-access article distributed under the terms of the Creative Commons Attribution License, which permits unrestricted use, distribution, and reproduction in any medium, provided the original author and source are credited. 